Sangeeta Mehta

Friederike Quittnat Pelletier

Maedean Brown

Cheryl Ethier

David Wells

Lisa Burry

Rod MacDonald

\section{Why substitute decision makers provide or decline consent for ICU research studies: a questionnaire study}

Received: 9 May 2011

Accepted: 13 October 2011

Published online: 26 November 2011

(C) Copyright jointly held by Springer and ESICM 2011

This article is discussed in the editorial available at:

doi:10.1007/s00134-011-2430-y.

Electronic supplementary material

The online version of this article (doi:10.1007/s00134-011-2411-1) contains supplementary material, which is available to authorized users.
S. Mehta $(\bowtie) \cdot$ F. Q. Pelletier ·

M. Brown - C. Ethier - D. Wells .

L. Burry · R. MacDonald

Department of Medicine and

Interdepartmental Division of Critical

Care Medicine, Mount Sinai Hospital,

600 University Ave, 18-216, Toronto,

ON M5G 1X5, Canada

e-mail: geeta.mehta@utoronto.ca

Tel.: +1-416-5864800

Fax: +1-416-5868480

L. Burry

Department of Pharmacy, Mount Sinai

Hospital, Toronto, ON, Canada
Abstract Purpose: Consent for research participation in the intensive care unit (ICU) is often obtained from a substitute decision maker (SDM). In this study we explored SDMs' reasons for declining or providing consent for research studies for critically ill adult family members.

Methods: Two questionnaires were developed, one directed at SDMs who agreed to have their relative participate in a research study (AGREE group), and another for SDMs who declined participation (DECLINE group). The questionnaires explored SDMs' opinions about research in general, timing of research approach, the informed consent process, and reasons for agreeing or declining participation. Results: Ninety-six SDMs completed the questionnaire (68 AGREE, 27 DECLINE). There were no differences between AGREE and DECLINE groups with respect to SDM demographics, perceived severity of illness of the patient, or the research study approach. The most common reasons for providing consent were potential for research to help others $(91 \%)$, research is important for medical progress $(88 \%)$, and trust in the medical team
(87\%). The most common reasons for declining consent were SDM was too anxious to consider research $(67 \%)$, fear that patient would receive experimental treatment $(37 \%)$, and concern about risks of the study (33\%). Conclusions: SDMs who agree to have a relative participate in an ICU research study are motivated by the potential benefit to the patient and altruism. SDMs who decline research participation, while not generally opposed to research, are fearful of study-related harm or discomfort for the patient, and are too anxious to consider a research study at that time.

Keywords Substitute decision makers - Clinical trials · Intensive care unit · Informed consent · Proxy consent

\section{Abbreviations \\ ICU Intensive care unit \\ RC Research coordinator \\ SDM Substitute decision maker}

\section{Introduction}

Obtaining informed consent for clinical research in the critical care setting is challenging. The majority of intensive care unit (ICU) patients are unable to provide consent because of sedation or altered level of consciousness. Thus, consent is usually obtained from the patient's legal representative or substitute decision maker 
(SDM), who according to the principle of substituted judgment is expected to consider and act on the patient's beliefs, leading to a decision which the patient would have made if he/she were capable [1,2]. As many ICU research studies require enrolment early in the course of the illness, the SDM may be approached soon after ICU admission, at a time when she/he is anxious, sleepdeprived, and overwhelmed by the large amount of medical information. Many other factors may also play a role in their decision-making, including personal research experience and relationships with the patient and healthcare team. Thus, for many reasons, the SDM may be unwilling or unable to make a decision regarding research, or may decline participation [3, 4].

Few studies have evaluated the decision-making of SDMs of adults who at baseline are competent to make their own decisions, and become incapable during acute illness. SDMs of these patients may be unprepared to make clinical or research decisions. Previous studies have focused on SDMs for non-critically ill children, and adults with dementia, both of whom lack capacity at baseline, and who are expected to require an SDM. In these patient groups, the next of kin may be more prepared to provide proxy consent. For parents of young children, the primary motives for consenting for research are future benefit for other children and the contribution to clinical science; benefit to their own child appears to be secondary $[5,6]$. For adult patients with dementia, the hope of benefit to the patient or patient's descendents predominated in drug trials, while more altruistic motives prevailed in studies not evaluating potential treatments [7].

The objectives of this study were to explore the motivations of SDMs for declining or providing consent for research studies for their critically ill adult family members; and to identify factors which may be associated with the response of SDMs, particularly those relating to research personnel and the approach for research, which are potentially modifiable. The results of this study will better inform the research community about the potential barriers and facilitators to the provision of informed consent, and help tailor our approach to family members of critically ill patients for research participation.

\section{Methods}

The study was conducted in the Mount Sinai Hospital ICU, a 16-bed adult medical-surgical ICU affiliated with the University of Toronto, between 2008 and 2010. The institutional research ethics board approved the study.

Please see the electronic supplementary material for additional methods relating to instrument development and questionnaire administration.

\section{Questionnaire construction}

Two separate questionnaires were developed to evaluate the motives of SDMs for providing or declining consent for a clinical research trial in the ICU. The questionnaires were tailored to specifically probe the reasons for the SDMs' decisions. The questionnaire for SDMs who agreed to have their relative participate in a research study (AGREE group) was comprised of 19 questions, and the questionnaire for SDMs who declined participation (DECLINE group) in a research study included 27 questions (Appendix 1, electronic supplementary material). Seventeen questions were identical in both questionnaires, relating to SDM's demographic information, the research study(ies) and timing of approach, the consenting process, and the number of family members involved in the decision-making. The questionnaires differed in the categorical options provided to SDMs regarding their opinion about research, and the reasons for providing or declining consent. This was because our literature search and group discussions identified very different reasons that SDMs provide or decline consent for research studies, with little overlap. In both questionnaires, we asked SDMs to provide a textual response to the question "What was the main reason for your decision about the research study?" and provided space for general textual feedback. The SDMs' textual responses to this question (Appendix 2, electronic supplementary material) were aggregated into groups representing similar themes; this was done by one investigator and adjudicated by a second. The questionnaire contained no identifying information regarding the patient or the SDM, other than the research study(ies) for which the SDM was approached.

\section{Questionnaire administration}

The target group was all SDMs who had been previously approached by the ICU research coordinator for possible participation in one or more parent clinical research studies ongoing in the ICU. Once the SDM had made a decision regarding the parent research study/studies, he/she was approached for questionnaire completion by a different research assistant, 24-72 $\mathrm{h}$ following the initial approach. This research assistant explained the rationale for the current study and the content of the questionnaire. Informed consent was implied by questionnaire completion. All successive SDMs were approached for questionnaire completion, unless the SDM did not speak English, if the patient had died, or if the SDM had provided consent for one study and declined another. If the SDM agreed to complete the questionnaire, he/she completed it anonymously, inserted it in an unmarked envelope, and returned it to an ICU staff member. 
Statistical analysis

The goal of this study was to obtain descriptive data, and to generate hypotheses. Thus we did not perform sample size calculation, and anticipated that approximately 100 respondents would allow us to achieve our goal $[8,9]$. Please see electronic supplementary material for a list of factors which we anticipated may be associated with consent provision and refusal. Categorical variables were expressed as percentages and continuous variables as mean and standard deviation (SD). Categorical variables were compared using the chi square test, and continuous variables using the Student's $t$ test. $p<0.05$ was considered significant.

\section{Results}

Overall, 113 SDMs were approached, and $84 \%$ in both groups $(N=95)$ completed the questionnaire, resulting in 68 in the AGREE group and 27 in the DECLINE group. Table 1 provides baseline characteristics of SDMs; overall demographics of both groups were similar. SDMs in both groups were primarily female. SDMs who

Table 1 Baseline characteristics of SDMs

\begin{tabular}{|c|c|c|c|}
\hline Characteristics & $\begin{array}{l}\text { Agree } \\
\text { group }\end{array}$ & $\begin{array}{l}\text { Decline } \\
\text { group }\end{array}$ & $p$ value \\
\hline SDMs approached $(N)$ & 81 & 32 & \\
\hline $\begin{array}{l}\text { Questionnaires completed } \\
{[N(\%)]}\end{array}$ & $68(84.0)$ & $27(84.4)$ & \\
\hline Age [years, median (range)] & $\begin{array}{l}50.5 \\
\quad(20-78)\end{array}$ & $\begin{array}{l}44.0 \\
\quad(28-74)\end{array}$ & 0.76 \\
\hline Male $[N(\%)]$ & $21(31.3)$ & $9(33.3)$ & 0.85 \\
\hline Ethnic group $[N(\%)]$ & & & 0.31 \\
\hline Asian & $7(10.3)$ & $6(22.2)$ & \\
\hline Caucasian & $51(75.0)$ & $18(66.7)$ & \\
\hline Other & $10(14.7)$ & $3(11.1)$ & \\
\hline Highest level of education & & & 0.21 \\
\hline High school & $24(35.3)$ & $8(29.6)$ & \\
\hline College diploma ${ }^{a}$ & $10(14.7)$ & $5(18.5)$ & \\
\hline Undergraduate degree $^{\mathrm{a}}$ & $15(22.1)$ & $4(14.8)$ & \\
\hline Graduate degree & $13(19.1)$ & $10(37.0)$ & \\
\hline Other & $2(2.9)^{\mathrm{b}}$ & & \\
\hline $\begin{array}{l}\text { English as first language } \\
{[N(\%)]}\end{array}$ & $58(85.3)$ & $17(63.0)$ & $<0.0001$ \\
\hline Relationship to patient $[N(\%)]$ & & & 0.19 \\
\hline Spouse/partner & $36(52.9)$ & $10(37.0)$ & \\
\hline Child & $16(23.5)$ & $10(37.0)$ & \\
\hline Parent & $10(14.7)$ & $2(7.4)$ & \\
\hline Sibling & $6(8.8)$ & $5(18.5)$ & \\
\hline $\begin{array}{l}\text { Health professional in family } \\
{[N(\%)]}\end{array}$ & $19(27.8)$ & $7(25.9)$ & 0.84 \\
\hline $\begin{array}{l}\text { a College diploma refers to a } 1 \\
\text { vocational institution or polytec } \\
\text { to a } 3 \text { - to } 4 \text {-year bachelors } \\
\text { university } \\
\text { b } 1 \text { SDM did not specify, and } 1\end{array}$ & $\begin{array}{l}\text { to } 2 \text {-yea } \\
\text { iic. Und }\end{array}$ & $\begin{array}{l}\text { gram } \\
\text { duate } \\
\text { by an }\end{array}$ & $\mathrm{ed} b$ \\
\hline
\end{tabular}

reported English as their first language were 7.25 times more likely (unadjusted odds ratio, 95\% CI 2.63, 20.0, $p<0.0001)$ to agree to study participation for their relative.

Table 2 summarizes details regarding the approach for research study(ies) by the primary research coordinator. A similar percentage of SDMs received a clinical update from a physician prior to the approach for research, but only a minority had the research study introduced by a physician during the clinical update. In 83.8 and $92.6 \%$ of the AGREE and DECLINE groups, respectively, the research study was introduced by the research coordinator $(p=0.617)$. The number of research studies presented to the SDM for consideration was significantly greater in the AGREE group (1.6 vs. $1.3, p=0.002$ ).

\section{AGREE group}

In the AGREE group 15\% $(N=10)$ of SDMs could not recall the theme of the primary research study. Table 3 presents textual responses provided by SDMs in response to the question "What was the main reason for your decision regarding the research study?" The two most common reasons cited were to help the patient recover faster $(N=17)$, and to improve patient care in the future $(N=14)$.

Figure 1 displays the factors which influenced the SDMs' decisions in the AGREE group. The four most common reasons for allowing their family member to participate were the research study will help others $(N=62,91 \%)$, research studies are important for medical progress $(N=60,88 \%)$, the SDM trusted the medical team $(N=59,87 \%)$, and the SDM's perception that the patient her/himself would have agreed to participate in the research study $(N=48,71 \%)$.

In response to the question "Would you agree to have your family member participate in a research study in the future?", of the 61 SDMs that responded, $51(83.6 \%)$ replied "Yes", 10 (16.4\%) replied "No", and 2 (3.2\%) replied "Maybe".

\section{DECLINE group}

In the DECLINE group 26\% $(N=7)$ of SDMs could not recall the theme of the primary research study. 92\% $(N=25)$ of SDMs agreed that the research coordinator spent enough time discussing the study, and provided the SDM sufficient time to make a decision; $96 \%$ agreed that the language used by the SDM was understandable. $37 \%$ $(N=10)$ were not aware that research studies are approved and monitored by independent research ethics boards. Table 4 presents textual responses provided by SDMs in response to the question "What was the main 
Table 2 Responses of SDMs regarding the primary research study approach

\begin{tabular}{|c|c|c|c|}
\hline Characteristics & $\begin{array}{l}\text { Agree group } \\
N=68\end{array}$ & $\begin{array}{l}\text { Decline group } \\
N=27\end{array}$ & $p$ value \\
\hline Received clinical update from physician before approach for RS $[N(\%)]$ & $40(58.8)$ & $11(40.7)$ & 0.27 \\
\hline Physician mentioned RS during clinical update $[N(\%)]$ & $6(8.8)$ & $0(0)$ & 0.14 \\
\hline Who first told SDM about the RS $[N(\%)]$ & & & 0.69 \\
\hline Research coordinator & $57(83.8)$ & $25(92.6)$ & \\
\hline Physician & $4(5.9)$ & 0 & \\
\hline Nurse & $2(2.9)$ & $1(3.7)$ & \\
\hline Social worker & $2(2.9)$ & 0 & \\
\hline Other & $3(4.4)$ & $1(3.7)$ & \\
\hline Patient's perceived severity of illness $[N(\%)]$ & & & 0.91 \\
\hline Critical & 44 (64.7) & $15(55.6)$ & \\
\hline Serious & $14(20.6)$ & $7(25.9)$ & \\
\hline Stabilized & $8(11.8)$ & $3(11.1)$ & \\
\hline Getting worse & $2(2.9)$ & $1(3.7)$ & \\
\hline Time from admission to research approach $[N(\%)]$ & & & 0.79 \\
\hline$<24 \mathrm{~h}$ & $28(41.1)$ & $13(48.1)$ & \\
\hline $24-48 \mathrm{~h}$ & $24(35.3)$ & $9(33.3)$ & \\
\hline$>48 \mathrm{~h}$ & $16(23.5)$ & $5(18.5)$ & \\
\hline Recollection of parent research study(ies) & $58(85.3)$ & $20(74.1)$ & 0.19 \\
\hline Number of studies presented to SDM [mean (range)] & $1.6(1-3)$ & $1.3(1-2)$ & 0.002 \\
\hline Number of relatives involved in consent process [mean (range)] & $1.9(1$ to $>4)$ & $1.7(1$ to $>4)$ & 0.65 \\
\hline $1[N(\%)]$ & $30(44.1)$ & $14(51.9)$ & \\
\hline 2 & $21(30.9)$ & $9(33.3)$ & \\
\hline 3 & $12(17.6)$ & $2(7.4)$ & \\
\hline$\geq 4$ & $5(7.4)$ & $2(7.4)$ & \\
\hline How decision about research participation was made $[N(\%)]$ & & & 0.20 \\
\hline SDM made the decision alone & $31(45.6)$ & $15(55.6)$ & \\
\hline Differing opinions, but family members established consensus & $9(13.2)$ & $1(1.5)$ & \\
\hline Family could not establish consensus, so SDM decided & 0 & $1(1.5)$ & \\
\hline All family members in agreement & $28(41.1)$ & $10(37.0)$ & \\
\hline Willingness to have family member participate in research study in the future & $51(83.6)^{\mathrm{a}}$ & $13(50.0)^{\mathrm{b}}$ & 0.001 \\
\hline
\end{tabular}

$R S$ research study

a 7 SDMs did not respond to this question

b 1 SDM did not respond to this question

Table 3 Major reason provided by SDM for agreeing to participate in the research study $(N=44)$

\begin{tabular}{lr}
\hline Responses & $N$ \\
\hline To help my loved one recover faster & 17 \\
To improve patient care in the future & 14 \\
Research is important to improve medical care & 9 \\
The research study was recommended by the physician & 1 \\
Study treatment is better than standard care & 1 \\
The research study was not invasive & 1 \\
The research study did not interfere with patient's primary care & 1
\end{tabular}

SDMs were asked to provide a textual response to the question "What was the main reason for your decision regarding the research study?" Responses were grouped into categories representing similar content. Verbatim responses are presented in Appendix 2, electronic supplementary material

reason for your decision regarding the research study?" The two most common reasons cited were fear of the research study causing harm or discomfort to the patient $(N=8,30 \%)$, and the SDM was too worried/anxious about the patient to consider research at the time $(N=6$, $22 \%)$.
Figure 2 displays the factors which influenced the SDMs' decisions in the DECLINE group. The four most common reasons for declining consent were the SDM was too worried to consider a research study $(N=18,67 \%)$, the SDM did not want the patient to receive an experimental treatment $(N=10,37 \%)$, the research study may adversely affect medical care $(N=9,33 \%)$, and there is more risk than benefit in the research study $(N=9,33 \%)$. Fewer than 5\% of SDMs agreed with the following statements: "science has no place in the ICU", "there was disagreement in my family about the study", "I have had bad experiences with research studies" and the "patient had been enrolled in a research trial in the past". No SDMs agreed with the statements "I do not trust medicine or research in general" and "I do not trust the medical team".

When the DECLINE group was asked "Would your decision be the same if you were asked today to have your relative participate in the research study?", $29 \%(N=7$ out of 24 who responded to this question) responded "No". Reasons cited for the change in their decision were the patient's condition was more stable $(N=6)$, the 
Fig. 1 SDMs' reasons for consenting for an ICU research study $(N=68)$

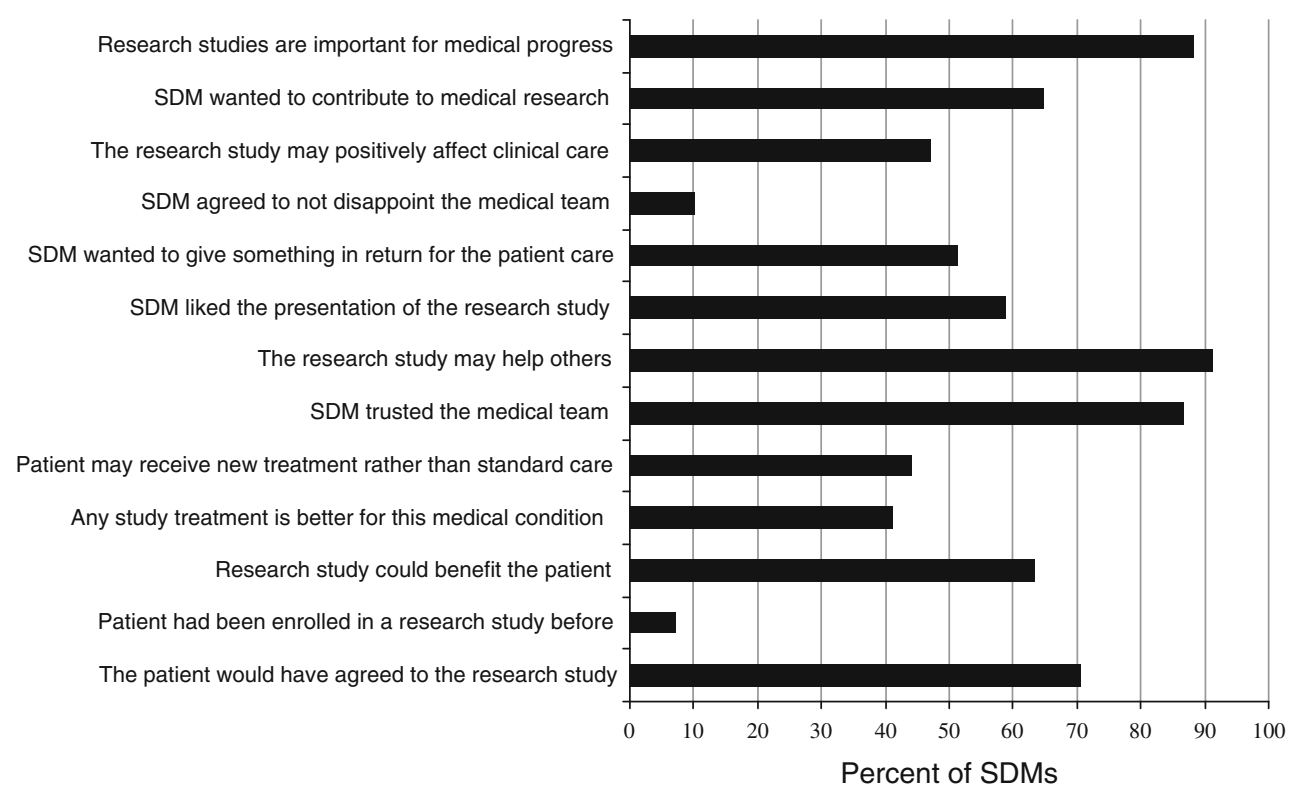

SDM = substitute decision maker
Table 4 Major reason provided by SDM for declining participation in the research study $(N=19)$

\begin{tabular}{ll}
\hline Responses & $N$ \\
\hline Fear of harm or discomfort to the patient & 8 \\
SDM too worried/anxious to consider research at that time & 6 \\
SDM was satisfied with the standard care provided & 2 \\
Personal reasons & 1 \\
Lack of discussion with attending physician regarding research & 1 \\
study & \\
Objection to study being sponsored by a pharmaceutical & 1 \\
company &
\end{tabular}

SDMs were asked to provide a textual response to the question "What was the main reason for your decision regarding the research study?" Responses were grouped into categories representing similar content; verbatim responses are presented in Appendix 2, electronic supplementary material

patient's condition was worse $(N=2)$, the SDM had more time to think about the study $(N=2)$, the SDM had received more information from ICU staff on the patient's condition $(N=1)$ and the research study $(N=1)$. When asked whether "Research studies are important for medical progress", $47 \%$ of SDMs $(N=9$ out of 19$)$ in the DECLINE group replied "Yes". In response to the question "Would you agree to have your family member participate in a research study in the future?", 50\% $(N=13$ out of 26) of the DECLINE group responded "Yes", compared with $83.6 \%$ of the AGREE group $(p=0.001)$.

\section{Discussion}

In this survey of SDMs approached for consent for ICU research studies, the most common reasons for providing consent were the potential for patient benefit, the potential to help future patients, and the importance of research for medical progress. The most common reason that SDMs declined research participation for their relative was that they were too worried about their loved one to consider a research study at the time of approach. This latter group was not opposed to research in principle, as reflected by $84 \%$ who completed the survey, $29 \%$ who would have agreed to research participation if they were approached later in the ICU course, $50 \%$ that would consent to research participation for their relative in the future, and $47 \%$ who agreed that research is important for medical progress. In our exploration of factors which may be associated with the SDM's decision, we found that SDMs who reported English as their first language were 7.25 times more likely to agree to study participation for their relative. It is not clear from our study whether this observation reflects poor understanding of the proposed research in SDMs whose first language was not English, or whether it reflects cultural or religious values relating to clinical research.

Our study revealed two notable findings about the process whereby SDMs make decisions about research participation for a loved one. First, we found that research decisions are often made collaboratively, with two or more relatives involved in the consent process in approximately $50 \%$ of cases. Thus, clinical investigators should encourage SDMs to have other family members 
Fig. 2 SDMs' reasons for declining consent $(N=27)$

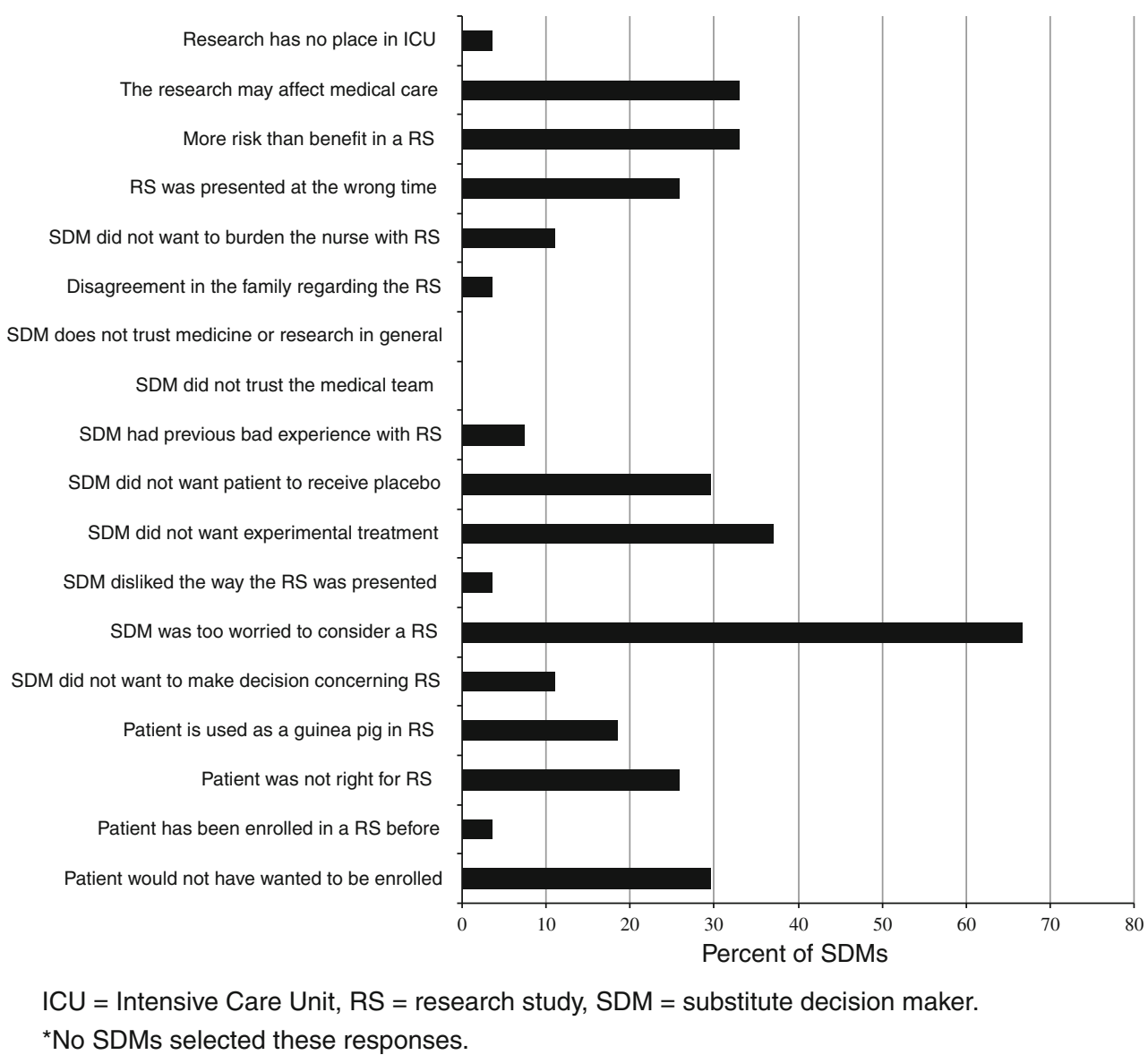

present during the consent discussion. Second, the potential for therapeutic misconception was highlighted by the SDMs in the AGREE group, who provided consent "To help my loved one recover faster". Therapeutic misconception arises wherever research subjects misunderstand the primary purpose of a clinical trial as therapeutic. This finding underscores the need for investigators to ensure that SDMs comprehend the distinction between the goals of clinical care and the goals of research, particularly in the ICU setting, where the SDM may be desperate to help their loved one.

While many previous studies have evaluated the experiences of SDMs relating to clinical decision-making for critically ill adults, few studies have explored the opinions of SDMs of adult patients regarding research participation in the ICU. Recently, two Canadian groups of investigators published work in this domain in abstract form. Within a prospective observational cohort study of research recruitment practices over a 1-month period in 23 adult ICUs, Burns et al. [10] probed SDMs' primary motive for consenting, and found that SDMs prioritized whether the patient would benefit from participation (65.9\%), over a desire to help others (16.3\%). Using structured interviews, Barrett and colleagues [11] surveyed 137 SDMs of critically ill adults in 4 ICUs regarding their role in research consent. While more than $85 \%$ of SDMs wished to be involved in research consent decisions, approximately $20 \%$ found the process very burdensome in the higher risk and shorter enrolment window scenarios, and up to $30 \%$ were very uncomfortable making the decision. Further, on the basis of the Hospital Anxiety and Depression Scale, more than $60 \%$ of SDMs were experiencing symptoms of anxiety related to the illness of their loved one. In our study, SDMs in the DECLINE group responded that anxiety regarding their loved one was an important factor in their decision. While we did not ask specifically about anxiety in the AGREE questionnaire, it may also be a factor in the decision of SDMs who provide consent for research. The possible influence of anxiety or apprehension about the patient, on research decision-making, should be further investigated.

Many SDMs have poor comprehension of information that is provided to them. A French survey of 76 SDMs of critically ill adult patients revealed that more than half failed to understand information presented to them regarding clinical diagnosis, prognosis, or treatment [8]. Chappuy et al. [9] reported similar poor comprehension in parents whose consent was sought for their child to participate in a therapeutic leukemia trial: half could explain neither the aim of the research nor the potential benefit of inclusion to their 
child. There is also evidence that research decision-making is burdensome, as Azoulay et al. [12] found that being asked to provide consent for research was associated with posttraumatic stress symptoms in $35 \%$ of family members of critically ill patients, compared with less than $10 \%$ in those involved in decisions about clinical care. Our data support these findings, in that a notable percentage of SDMs, particularly in the DECLINE group, were unable to recall the theme of the parent study for which they were approached, and many responded that they were too anxious about their loved one to consider research. The findings of our study and others suggest that SDMs may experience difficulty processing large amounts of information in the face of critical illness in a loved one; and while they necessarily must make treatment decisions for their loved ones, decisions about research are not perceived similarly $[10,12]$.

Strengths of our study include a comprehensive anonymous questionnaire, the inclusion of both SDMs who provided and declined research consent, an excellent response rate in both groups, and the surveying of SDMs early after the approach for the parent study, in order to reduce possible recall bias. In addition, to reduce response bias, a research assistant who was not involved in the primary consenting process approached the SDMs for questionnaire completion. Finally, the use of two different questionnaires for SDMs who provided and declined consent provided us with information specific to the two groups of SDMs.

There are several limitations of our study. We did not use a previously validated questionnaire, since there are no published questionnaires which encompassed all of the variables of interest. Secondly, self-report questionnaires have multiple inherent limitations, including recall bias, non-response bias, and completion bias, compromising the generalizability of results. Instrumentation bias is also a potential issue, given that we provided a list of suggested reasons for the SDMs' decision, and the response options differed in the AGREE and DECLINE questionnaires. However we invited SDMs to provide additional reasons which we had not listed, in the form of a textual response, which we aggregated into thematic groups (Appendix 2, electronic supplementary material). We recognize that this thematic grouping may be biased, and reflects the inherent limitations of qualitative research and content analysis in particular. This was a single-centre questionnaire, and the results may not be generalizable to SDMs in other institutions. In addition, our study sample size was inadequate to demonstrate differences in many group characteristics; a larger sample may have identified other factors associated with the SDMs' response. While the lack of recall of SDMs for the parent study may be indicative of anxiety or apprehension, we cannot exclude the possibilities that the research coordinator failed to effectively impart information regarding the clinical research trial, or that comprehension was poor in SDMs whose first language was not English. Finally, it is possible that the degree of invasiveness of the parent study(ies) influenced the SDM's decision; however, we are unable to confirm or refute this, because we did not record the proposed studies, and because all of the SDMs were approached for at least one invasive study and a second observational or less-invasive study.

\section{Conclusions}

Our study provides a unique perspective of the SDM experience of research decision-making on behalf of critically ill relatives. In summary, SDMs' major motivations when they agree to have a critically ill relative participate in a research study are potential patient benefit, altruism, and the desire to advance medical progress. SDMs who decline are not opposed to research in principle, but are too apprehensive about their loved ones to consider research.

Conflict of interest None of the authors has declared any financial or non-financial competing interests.

\section{References}

1. Luce JM (2003) Is the concept of informed consent applicable to clinical research involving critically ill patients? Crit Care Med 31(3 Suppl):S153-S160

2. Luce JM, Cook DJ, Martin TR, Angus DC, Boushey HA, Curtis JR, Heffner JE, Lanken PN, Levy MM, Polite PY, Rocker GM, Truog RD (2004) The ethical conduct of clinical research involving critically ill patients in the United States and Canada: principles and recommendations. Am J Respir Crit Care Med 170:1375-1384
3. Ciroldi M, Cariou A, Adrie C, Annane D, Castelain V, Cohen Y, Delahaye A, Joly LM, Galliot R, Garrouste-Orgeas M, Papazian L, Michel F, Barnes NK, Schlemmer B, Pochard F, Azoulay E, Famirea study group (2007) Ability of family members to predict patient's consent to critical care research. Intensive Care Med 33:807-813

4. Coppolino M, Ackerson L (2001) Do surrogate decision makers provide accurate consent for intensive care research? Chest 119:603-612
5. Van Stuijvenberg M, Suur MH, De Vos S, Tjiang GCH, Steyerberg EW, Derksen-Lubsen E, Moll HA (1998) Informed consent, parental awareness, and reasons for participating in a randomised controlled study. Arch Dis Child 79:120-125

6. Sammons HM, Atkinson M, Choonara I, Stephenson T (2007) What motivates British parents to consent for research? A questionnaire study. BMC Pediatr $7: 12$ 
7. Sugarman J, Cain C, Wallace R, WelshBohmer KA (2001) How proxies make decisions about research for patients with Alzheimer's disease. J Am Geriatr Soc 49:1110-1119

8. Azoulay E, Chevret S, Leleu G, Pochard F, Barboteu M, Adrie C, Canoui P, Le Gall JR, Schlemmer B (2000) Half the families of intensive care unit patients experience inadequate communication with physicians. Crit Care Med 28:3044-3049

9. Chappuy H, Baruchel A, Leverger G, Oudot C, Brethon B, Haouy S, Auvrignon A, Davous D, Doz F, Tréluyer JM (2010) Parental comprehension and satisfaction in informed consent in paediatric clinical trials: a prospective study on childhood leukaemia. Arch Dis Child 95:800-804
10. Burns KEA, Zubrinich C, Tan W, Smith O, McDonald E, Skrobik Y, Saginur R, Jeffs L, Marshall J, Heslegrave R, Rubenfeld GD, Cook DJ (2010) Understanding barriers to recruitment and why patients and surrogates consent for Critical Care research: The Consent Study. Am J Respir Crit Care Med 181:A6047

11. Barrett K, Athaide V, Smith OM, McDonald E, Pinto R, Stevenson J, Go S, Elman J, Friedrich JP, Cook DJ, Scales DC, Ferguson ND, the Canadian Critical Care Trials Group (2009) Proxy evaluations rating methods for inclusion into trials (PERMIT 2). Am J Respir Crit Care Med 179:A1573
12. Azoulay E, Pochard F, Kentish-Barnes $\mathrm{N}$, Chevret S, Aboab J, Adrie C, Annane D, Bleichner G, Bollaert PE, Darmon M, Fassier T, Galliot R, Garrouste-Orgeas M, Goulenok C, Goldgran-Toledano D, Hayon J, Jourdain M, Kaidomar M, Laplace C, Larche J, Liotier J, Papazian L, Poisson C, Reignier J, Al Saidi F, Schlemmer B (2005) Risk of post-traumatic stress symptoms in family members of intensive care unit patients. Am J Respir Crit Care Med 171:987-994 